Manuelle Medizin 2015 · 53:302

DOI 10.1007/s00337-015-0002-x

Online publiziert: 25 . Juli 2015

(c) Springer-Verlag Berlin Heidelberg 2015

\author{
W. König \\ Bad Aibling, Deutschland
}

\title{
Themenheft "Musikermedizin" als Beispiel für die Entstehung funktioneller Schmerzen
}

verschwinden die Schmerzen zu einem sehr hohen Anteil erst, wenn die Einschränkung behoben ist. Nur ein passiv frei bewegliches Gelenk ermöglicht es der aktiven, dann nicht mehr gestörten Steuerung, den Kopf optimal zu zentrieren. Diese Zusammenhänge sind $\mathrm{zu}$ beobachten, lange bevor die strukturellen Zerstörungen einsetzen, Magnetresonanztomogramm und Röntgenbild Veränderungen zeigen und Operationen geplant werden. Eine korrekte zervikothorakale Haltung, ein stabil fixiertes Schulterblatt und ein frei bewegliches Schultergelenk sind die beste Prophylaxe für ein Impingement-Syndrom, das mittlerweile sehr verbreitet auftritt.

Bei der wissenschaftlichen Bearbeitung dieses Themenkomplexes wird man sich aber auch einem wesentlichen Problem stellen müssen. Was immer man therapiert (manualmedizinisch/ osteopathisch, physiotherapeutisch etc.) - der Erfolg liegt immer in der Berücksichtigung und dem Eingehen auf die individuellen Befunde. Hieraus ergibt sich ein grundsätzliches Konfliktpotenzial mit randomisierten Studien. Standardisierte Therapien sind nicht so erfolgreich, individualisierte Therapien sind nicht so wissenschaftlich basiert. Für mich ist es außerordentlich erstaunlich, dass dieser Konflikt bisher nicht weiter thematisiert wurde. Die wissenschaftliche Aufarbeitung unserer Arbeitsweise ist unumgänglich. Aber dazu gehören auch die kritische Würdigung der eingesetzten Instrumente und nicht nur die Nachahmung der Arbeitsweise anderer Fachbereiche. Ich hoffe, dass die systematische Aufarbeitung des Themas "funktioneller Schmerz" eine kreative Fortsetzung findet, denn hier sehe ich eine bedeutsame Möglichkeit zur Prophylaxe vieler Erkrankungen des Bewegungsapparats.

\section{Korrespondenzadresse}

\section{W. König}

Rennbahnstr. 2, 83043 Bad Aibling,

Deutschland

hwkoenig@gmx.de 